\title{
EDITORIAL
}

\section{The Evidence Is at Hand for Improving Care in Settings Where Residents Train}

\author{
David P. Stevens, MD', Constance S. Sixta, PhD, RN, MBA'², Ed Wagner, MD, MPH', \\ and Judith L. Bowen, $M D^{4}$
}

'Quality Literature Program, Dartmouth Institute for Health Policy and Clinical Practice, Lebanon, NH, USA; ${ }^{2}$ School of Nursing, University of Texas Health Science Center, Houston, TX, USA; ${ }^{3}$ MacColl Institute for Healthcare Innovation, Group Health Center for Health Studies, Seattle, WA, USA; ${ }^{4}$ Department of Medicine, Division of General Internal Medicine \& Geriatrics, Oregon Health \& Science University, Portland, OR, USA.

J Gen Intern Med 23(7):1116-7

DOI: $10.1007 / \mathrm{s} 11606-008-0674-1$

(C) Society of General Internal Medicine 2008

$\mathrm{S}$ even years ago the Institute of Medicine's report, Crossing the Quality Chasm ${ }^{1}$, issued a challenge to transform America's healthcare system. If this challenge is to be met any time soon, it will require profound leadership by general internists. This, in turn, requires that the next generation of internists be trained in residency programs with a commitment to transforming healthcare in the settings where those future physicians train and practice. In 1999, the Accreditation Council for Graduate Medical Education (ACGME) radically redefined physician competence by framing two of the six core physician competencies as system redesign and improvement-SystemsBased Practice (SBP) and Practice-Based Learning and Improvement (PBLI). ${ }^{2}$ The American Board of Medical Specialties adopted the same competencies for specialty certification, thus acknowledging the importance of the continuum of a physician's learning. These two competencies redefined healthcare and health professions' education by acknowledging physicians' professional responsibility to improve healthcare and systems.

Batalden and Davidoff ${ }^{3}$ have taken this point further by arguing that continuous improvement of patient care, healthcare systems, and health profession education-all three-must be inseparably linked if healthcare improvement is to be sustained. They postulate that improving both healthcare outcomes and education is part of an entire professional career in healthcare, not just during the formal educational components of early education and training.

A critical literature review of educational strategies addressing healthcare improvement ${ }^{4}$ found that only 4 of 39 qualityimprovement educational initiatives linked improved education with improved patient care. In the past decade, a number of performance improvement initiatives have helped practicing internal medicine physicians build effective systems of chronic illness care. During that time, however, little has been done to transform healthcare systems that serve as the learning environment for internal medicine residents. The current volume of The Journal of General Internal Medicine provides an optimistic note by reporting a spectrum of initiatives that explicitly address patient care and health system improvement in settings where residents train. The articles range from strategic planning for quality improvement activities to the implementation of substantial program redesign. They reflect the complexity of initiating change in academic healthcare systems, but they also illustrate both the value and the feasibility of education investigations that demonstrate simultaneous improvements in both education and patient care. ${ }^{5}$

We observed similar diversity of initiatives and outcomes in the over 50 residency training settings in academic health centers that participated in the Academic Chronic Care Collaborative (ACCC) and the California Academic Chronic Care Collaborative (CA ACCC). ${ }^{6}$ Each of these training programs endeavored to implement the Chronic Care Model, an evidence-based strategy to improve chronic illness care, ${ }^{7}$ while redesigning residency training in the care of persons with chronic conditions. Not surprisingly, training programs found it generally easier to improve process measures_-both educational and clinical-than to achieve significant improvement in clinical outcomes. The profound achievements reported by training programs in this issue of JGIM demonstrate that substantial and measurable practice improvement linked to educational redesign is possible.

One of the current reports reminds us of the substantial variation in practice and the critical need for systematic improvement. Mladenovic and colleagues ${ }^{8}$ enlisted site champions at 23 internal medicine residency program continuity practices to abstract nearly 5,000 patient records and provide practice system characteristics. On average, patients received only $57 \%$ of the recommended preventive cardiology recommendations, leaving plenty of opportunity for improvement in care delivery and, we presume, education.

Three of the current reports describe implementation of improvement strategies in the ambulatory setting. Warm and colleagues $^{9}$ report the impact of a transformational redesign of an internal medicine residency training program. Innovations included a year-long continuous ambulatory group practice experience and implementation of the chronic care modelnotably including both training in quality improvement strategies and practice in multidisciplinary teams. The redesign resulted in significant improvement in both care process and clinical outcome measures.

Dearinger and colleagues ${ }^{10}$ demonstrated the benefit of resident continuity on the outcomes of patients with diabetes. Continuity with the same resident physician produced a significant decrease in hemoglobin Alc levels compared to patients who received care from multiple different resident physicians.

Oyler and colleagues ${ }^{11}$ report a residency redesign that supports residents in their implementation of positive system change during ambulatory rotations. The curriculum integrated training in quality improvement principles, assessment of the 
status of clinical guideline implementation through chart review, selection of a quality problem, and the use of the American Board of Internal Medicine Performance Improvement Module ${ }^{12}$ to plan and implement performance improvement, demonstrating measurable immediate results.

Peters and colleagues ${ }^{13}$ describe a different strategy, namely the use of a self-directed web-based 4-week curriculum that incorporated content that could not be taught by current faculty. Residents participating in the curriculum had significantly improved knowledge and self-assessed competency as compared to controls, although there was no significant improvement in attitude toward SBP/PBLI competencies. This work suggests that, in the absence of local faculty expertise, important content can be taught effectively using a self-study medium with improved knowledge and self-perceived competency.

What can be learned from these reports of substantial residency redesign focused on SPB and PBLI? First, they build confidence that a commitment to redesign of care can be linked successfully to explicit educational strategies. Second, the predominance of reports from ambulatory settings begs the question: Do ambulatory environments provide better opportunities for redesign? Perhaps the microsystems that comprise ambulatory practice lend themselves more readily to administrative control than inpatient settings where the complexity, pace, and acuity of illness often dominate the ability of program directors and others to craft reliable systems for improvement. Finally, these reports suggest characteristics of successful early adopter institutions. Common themes include strong, innovative program leadership; visionary support from institutional leaders reflected in the investment of precious financial resources in spite of a resource-constrained healthcare and educational system; an institutional culture that embraces continuous improvement; commitment to inter-professional practice across practice settings; and-perhaps most essential for sustaining successcommitment to re-thinking the role of the internist as clinical leader for improving patient care outcomes, system performance, and health profession education.
Corresponding Author: David P. Stevens, MD, Quality Literature Program, Dartmouth Institute for Health Policy and Clinical Practice, 30 Lafayette Street, Lebanon, NH 03766, USA (e-mail: David.P. Stevens@Dartmouth.edu).

\section{REFERENCES}

1. Institute of Medicine. Crossing the quality chasm: a new health system for the twenty-first century. Washington, DC: The National Academy Press; 2001

2. Batalden P, Leach D, Swing S, Dreyfus H, Dreyfus S. General competencies and accreditation in graduate medical education. Health Affairs. 2002;21:103-11.

3. Batalden PB, Davidoff F. What is "quality improvement" and how can it transform health care. Qual Saf Health Care. 2007;16:2-3.

4. Boonyasai RT, Windish DM, Chakraborti C, Feldmon LS, Rubin H, Bass EB. Effectiveness of teaching quality improvement to clinicians. JAMA. 2007;298:1023-37.

5. Stevens DP, Andersson-Gare B. A proposal: Publication guidelines for healthcare improvement education reports. Quality and Safety in Health Care. 2007;16:402-3.

6. Stevens DP, Wagner EH. Transform residency training in chronic illness care-now. Acad. Med. 2006;81:685-7.

7. Wagner EH, Austin BT, David C, Hindmarsh M, Schaefer J, Bonomi A. Improving chronic illness care: Translating evidence into action. Health Affairs. 2001;20:64-78.

8. Mladenovic J, Shea JA, Duffy D, Lynn LA, Holmboe ES, Lipner RS. Variation in internal medicine residency clinic practices: assessing practice environments and quality of care. J Gen Intern Med. 2005;20:1181-87.

9. Warm EJ, Schauer DP, Diers T, Mathis BR, Neirouz Y, Boex JR, Rouan GW. The ambulatory long-block: An Accreditation for Graduate Medical Education (ACGME) Educational Innovations Project (EIP). J Gen Int Med. 2008; in press.

10. Dearinger AT, Wilson JF, Griffith CH, Scutchfield FD. The effect of physician continuity on diabetic outcomes in a resident community clinic. J Gen Int Med. 2008; in press.

11. Oyler J, Vinci L, Arora V, Johnson J. Teaching internal medicine residents quality improvement techniques using ABIM's Practice Improvement Modules. J Gen Int Med. 2008; in press.

12. http://www.abim.org/pims/default.aspx (accessed May 6, 2008).

13. Peters AS, Kimura J, Ladden MD, March E, Moore GT. A selfinstructional model to teach systems-based practice and practice-base learning and improvement. J Gen Int Med. 2008; in press. 